Special Issue of the 6th International Congress \& Exhibition (APMAS2016), Maslak, Istanbul, Turkey, June 1-3, 2016

\title{
In Situ Solution Process for Fabricating Thermally and Mechanically Stable Highly Conductive ZnO-CNT Fiber Composites
}

\author{
M.M. HOSSAIN ${ }^{a}$, S.B. SON ${ }^{b}$ AND J.R. HAHN ${ }^{a, c, *}$ \\ ${ }^{a}$ Department of Chemistry and Bioactive Material Sciences, Research Institute of Physics and Chemistry, \\ Chonbuk National University, Jeonju 561-756, Korea \\ ${ }^{b}$ Jeonju Center, Korea Basic Science Institute, Jeonju 561-756, Republic of Korea \\ ${ }^{c}$ Textile Engineering, Chemistry and Science, North Carolina State University, \\ 2401 Research Dr., Raleigh, NC 27695-8301, USA
}

\begin{abstract}
A simple in situ solution process was developed to produce a mechanically and thermally stable $\mathrm{ZnO}$-carbon nanotube fiber composite. $\mathrm{ZnO}$ nanoparticles were homogeneously deposited onto the surfaces of and interstices within CNT fibers (between individual CNTs). X-ray photoelectron spectroscopy and Raman analysis revealed that $\mathrm{ZnO}$ nanoparticles contained oxygen vacancy defects and CNT fibers included oxygen containing functional group that strongly interacted with $\mathrm{Zn}$. The strong interaction enhanced the mechanical properties of the composite fibers. The Young modulus (20 GPa) and tensile strength $(118 \mathrm{MPa})$ were enhanced compared to the corresponding values of the pristine CNT fibers. The thermal stability was high up to $880^{\circ} \mathrm{C}$ and light absorption was enhanced across the UV to near IR region in a $\mathrm{ZnO}-\mathrm{CNT}$ fiber composite. The electrical conductivity of the composite was high up to $954 \mathrm{~S} / \mathrm{cm}$ despite semiconductor deposition.
\end{abstract}

DOI: 10.12693/APhysPolA.131.124

PACS/topics: 81.05.-t

\section{Introduction}

Fiber-shaped materials show promise as energy storage candidates. For example, woven textiles prepared from fiber-shaped solar cells or supercapacitors can provide a high degree of flexibility and low weight while offering solar absorption capabilities for electricity generation and a high conductivity for photoexcited electrons. Fiber-shaped materials are useful during electricity interruption, particularly in harsh environments. The use of fiber-shaped materials in flexible, lightweight and portable electronic devices has been extensively explored [16]. Fiber-shaped materials such as Kevlar [1], metal fibers [2], metal wires [3], carbon fibers [4], carbon nanotubes (CNTs) [5], or graphene [6] fiber have been explored for their utility. Polymer fibers have enabled significant breakthroughs in the fabrication of flexible devices although they tend to provide poor electrical conductivity or insulation properties $[7,8]$. Metal wires have been explored as efficient electrodes in electronic devices due to their high electrical conductivities [9]; however, metal wires tend to be heavy, offer poor flexibility, and cannot be effectively woven into textiles.

The excellent mechanical and electrical conductivity properties $[5,10,11]$ of CNT fibers have been widely studied. Dry or wet spinning processes [12] have been used to spin CNTs into fibers hundreds of meters in length and

*corresponding author; e-mail: jrhahn@jbnu.ac.kr a few micrometers in width CNT fibers tend to exhibit relatively low electrocatalytic activities, which presents a significant barrier to their electronic applications. Furthermore, CNT fiber devices offer low efficiency photoelectric conversion and energy storage properties [5, 13]. The deposition of a variety of metal oxides $\left(\mathrm{MnO}_{2}\right.$ [14], $\mathrm{NiO}$ [15], $\mathrm{RuO}_{2}$ [16], $\mathrm{V}_{2} \mathrm{O}_{5}$ [17], $\mathrm{TiO}_{2}$ [3], $\mathrm{ZnO}$ [18]) or metal nanoparticles (Pt [19], PtRu [20], PtSn [21], $\mathrm{Pd}[22], \mathrm{Au}[22], \mathrm{Ru}[23], \mathrm{Ag}[24])$ onto the CNT fiber surfaces has been reported to improve the electrochemical and optical properties of CNT fibers. Metal oxides or metal nanoparticles are typically deposited randomly and inhomogeneously onto CNT fibers. The smooth deposition of metal or metal oxide nanoparticles is necessary for enhancing the electronic and optical properties of CNT fibers. Smooth deposition requires CNT pretreatment, CNT functionalization, CNT acid oxidation, surfactant introduction, or particular fabrication processes [19-24]. Unfortunately, the electrical conductivity of a CNT fiber typically decreases upon deposition of semiconductive nanoparticles onto the CNT surfaces.

Here we developed an in situ solution process for CNT fiber modification with $\mathrm{ZnO}$ nanoparticles. We previously reported the fabrication of $\mathrm{ZnO} / \mathrm{C}$ core-shell nanorods through an in situ process [25, 26]. We modified that method to fabricate $\mathrm{ZnO}-\mathrm{CNT}$ fiber composites that exhibited excellent mechanical and light absorption properties and a high electrical conductivity. $\mathrm{ZnO}$ was chosen because it is low in cost, environmentally friendly, provides strong photoinduced hole oxidation, and has a high electron mobility [27]. By incorporating $\mathrm{ZnO}$ into 
CNT fibers, we enhanced the mechanical properties and electrical conductivity of the fibers. Our in situ method was performed without the need for pretreatment, oxidation, or CNT functionalization steps. The individual CNTs and $\mathrm{ZnO}$ nanoparticles strongly interacted after thermal decomposition of a zinc acetate layer on the CNT fiber surfaces. The strong interaction enabled the smooth deposition of $\mathrm{ZnO}$ nanoparticles, enhanced the Young modulus and stress, enabled light absorption throughout the 300-2100 $\mathrm{nm}$ region and enhanced the thermal stability of the CNT fibers as compared to the pristine CNT fibers. The electrical conductivity did not decrease significantly upon $\mathrm{ZnO}$ deposition due to the strong interactions between $\mathrm{Zn}$ and the CNTs.

\section{Experimental}

\subsection{Chemicals}

Zinc acetate dihydrate (Sigma Aldrich, 99\% purity) was used as a precursor material for the $\mathrm{ZnO}$ nanoparticles. Ethanol (Sigma Aldrich, 95\%) was used as a solvent to prepare the zinc acetate dihydrate solution. Au wires (0.05 mm in diameter, 171105, Nilaco Co) and silver paste (Leit Silver, Sigma Aldrich) were used to measure the electrical conductivity of the CNT fibers and the $\mathrm{ZnO}-\mathrm{CNT}$ fiber composite. CNT fibers were fabricated from acetone (99.5\%, Daejung Chemicals and Metals), ferrocene (98\% Sigma Aldrich) and thiophene (99\%, Sigma Aldrich). The CNT fibers were concentrated using dimethyl sulfoxide (DMSO, 99.5\%, Sigma Aldrich).

\subsection{Instruments}

Bath sonication was performed to prepare the zinc acetate dihydrate solution. The electrical conductivities of the CNT fibers and composite fibers were measured using a four-point probe combined with Keithley Instruments (2400 SourceMeter and 2000 MULTIMETER). The sample structures were analyzed using field emission scanning electron microscopy (FESEM, S4700, Hitachi) and X-ray diffraction (XRD, Smart Lab, Rigaku). The samples were spectroscopically characterized by UV/vis/NIR spectrophotometry (V670, JASCO), the Raman spectroscopy (HORIBA JOBIN YVON, Lab RAM HR, Laser 514.54 and 632.817), and X-ray photoelectron spectroscopy (XPS, K-Alpha, Thermo Scientific). The mechanical properties were measured using a Universal Testing Machine (UTM 5567A, INSTRON). Focused ion beam (FIB) techniques were used to cut the CNT fibers, and energy dispersive X-ray (EDX) characterizations were performed using a HELIOS NANOLAB 650 (FEI).

\subsection{Preparation of the CNT fibers}

A liquid feedstock [28] containing acetone (a carbon source), ferrocene ( $0.2 \mathrm{wt} \%$, a catalyst precursor), and thiophene $(0.8 \mathrm{wt} \%$, a promoter $)$ was injected into a heated furnace at $1200^{\circ} \mathrm{C}$. The carrier gas was delivered at a rate of 1000 SCCM to synthesize continuous CNT fibers, as described previously [28]. The resulting continuous CNT array was then declined into a water tank and subsequently wound at a rate of $5 \mathrm{~m} / \mathrm{min}$. The CNT fibers were densified in DMSO followed by drying at $100^{\circ} \mathrm{C}$.

\subsection{Fabrication of a $\mathrm{ZnO}-C N T$ fiber composites in situ}

CNT fibers were mounted on a frame of stainless steel wire (Fig. 1a). Zinc acetate dihydrate $(3 \mathrm{~g})$ was dissolved in ethanol (50 ml, Fig. 1e) and sonicated for $1 \mathrm{~h}$ to produce a homogeneous solution $(60 \mathrm{mg} / \mathrm{ml})$ at room temperature. CNT fibers on the steel frame were positioned in a Petri dish (Fig. 1b), and the zinc acetate dihydrate solution was poured slowly into the dish. As crystals of zinc acetate formed in the solution (Fig. 1d), the steel frame was quickly removed from the solution and dried at $180^{\circ} \mathrm{C}$ in a preheated furnace for 2 min to remove the ethanol solvent. The zinc acetate dihydrate solution in the dish was poured into a vial and sonicated again to dissolve any solid zinc acetate precipitate. As the solution became clear, the dried CNT fiber frame was placed into the solution again. A total of 3 or 4 cycles were applied to deposit the zinc acetate onto the CNT fiber surface. During each cycle, the fibers were immersed into the solution for 3 to $5 \mathrm{~min}$. Long immersion times could permit the zinc acetate solution to penetrate the CNT fibers. It was important to avoid rapid precipitation onto the CNT fiber surfaces, however, as opposed to penetration of the fibers over long immersion times. A low lab temperature $\left(10-15^{\circ} \mathrm{C}\right)$ facilitated the effective penetration of the solution into the CNT fibers. Finally the zinc acetate deposited CNT fibers were placed in a preheated furnace $\left(200^{\circ} \mathrm{C}\right)$ and the temperature was slowly increased to $450{ }^{\circ} \mathrm{C}$ at a rate of $4^{\circ} \mathrm{C} / \mathrm{min}$. The fibers were then heated at $450^{\circ} \mathrm{C}$ for $20 \mathrm{~min}$ to decompose the zinc acetate to produce the $\mathrm{ZnO}-\mathrm{CNT}$ fiber composite. Other samples of $\mathrm{ZnO}-\mathrm{CNT}$ fiber composites were prepared at 350,400 , and $500^{\circ} \mathrm{C}$ to investigate the effects of the temperature on the sample structure.

\subsection{Fabrication of $\mathrm{ZnO}-\mathrm{CNT}$ fiber composites ex situ}

A zinc acetate solution (50 $\mathrm{ml}, 60 \mathrm{mg} / \mathrm{ml}$ in ethanol) in a round-bottom flask was stirred in a silicon oil bath for $2 \mathrm{~h}$ at $100^{\circ} \mathrm{C}$ to produce $\mathrm{ZnO}$ nanoparticles. The CNT fibers mounted on a steel frame were immersed in a solution of $\mathrm{ZnO}$ nanoparticles for $30 \mathrm{~min}$ to produce a $\mathrm{ZnO}-\mathrm{CNT}$ fiber composite. The composite was then removed and dried at $100^{\circ} \mathrm{C}$ for $2 \mathrm{~h}$ to evaporate away the ethanol solvent.

\subsection{Synthesis of the purified $\mathrm{ZnO}-\mathrm{CNT}$ fiber composites}

CNT fibers mounted on a glass slide were heated at $400{ }^{\circ} \mathrm{C}$ for 45 min to oxidize any metal impurities. After cooling, the heated CNT fibers were immersed in concentrated $\mathrm{HCl}$. The $\mathrm{CNT}$ fibers in $\mathrm{HCl}$ were boiled at $80^{\circ} \mathrm{C}$ for $4 \mathrm{~h}$ to remove metal impurities as metal chlorides. After boiling, the fibers were washed in DI water several 


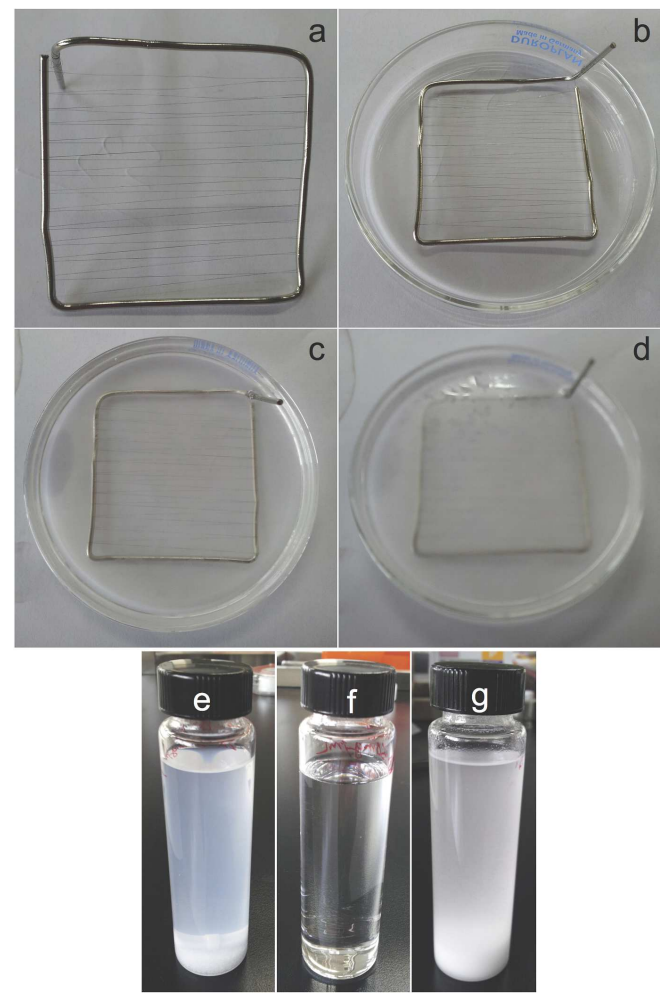

Fig. 1. (a) CNT fibers mounted on a frame of stainless steel wire. (b) CNT fibers on the frame were positioned in a Petri dish. (c) CNT fibers frame immersed in $60 \mathrm{mg} / \mathrm{ml}$ solution of zinc acetate. (d) Crystals of zinc acetate formed in the solution. (e) Zinc acetate in ethanol. (f) Zinc acetate solution after bath sonication. (g) Zinc acetate solution $(60 \mathrm{mg} / \mathrm{ml})$ after settling down for a few minutes.

times to remove any remaining impurities and were heated at $400^{\circ} \mathrm{C}$ for $45 \mathrm{~min}$. Finally, the purified CNT fibers were used to produce a $\mathrm{ZnO}-\mathrm{CNT}$ fiber composite using the procedure described in Sect. 2.4.

\section{Results and discussion}

Figures $2 \mathrm{a}-2 \mathrm{~d}$ show FESEM images of the $\mathrm{ZnO}-\mathrm{CNT}$ fiber composites prepared on a Si wafer. Several CNT fibers are shown in Fig. 2a. The $\mathrm{ZnO}$ nanoparticles are apparent in the magnified views of the regions marked with rectangles (Fig. 2c,d). ZnO nanoparticles were deposited homogeneously with an average size of $50 \mathrm{~nm}$. The aggregated $\mathrm{ZnO}$ nanoparticles on the CNT fibers appeared as large spheres in Fig. 2b and c. As described in Sect. 2, the $\mathrm{ZnO}-\mathrm{CNT}$ fiber composites were synthesized from assynthesized or purified CNT fibers. For comparison, FESEM images of the as-synthesized or purified CNTs were obtained, as shown in Fig. 2e and f, respectively. Purification was performed by heating and $\mathrm{HCl}$ treatment to remove metal impurities from CNT fiber. FESEM images of the as-synthesized CNT fibers revealed individual CNTs and clearly differed from the surface images of the
ZnO-CNT fiber composites. After purifying the CNT fibers, the morphologies were characterized and found to be similar to those of the as-synthesized fibers, indicating that the CNT fibers were not damaged through heating and $\mathrm{HCl}$ treatment.

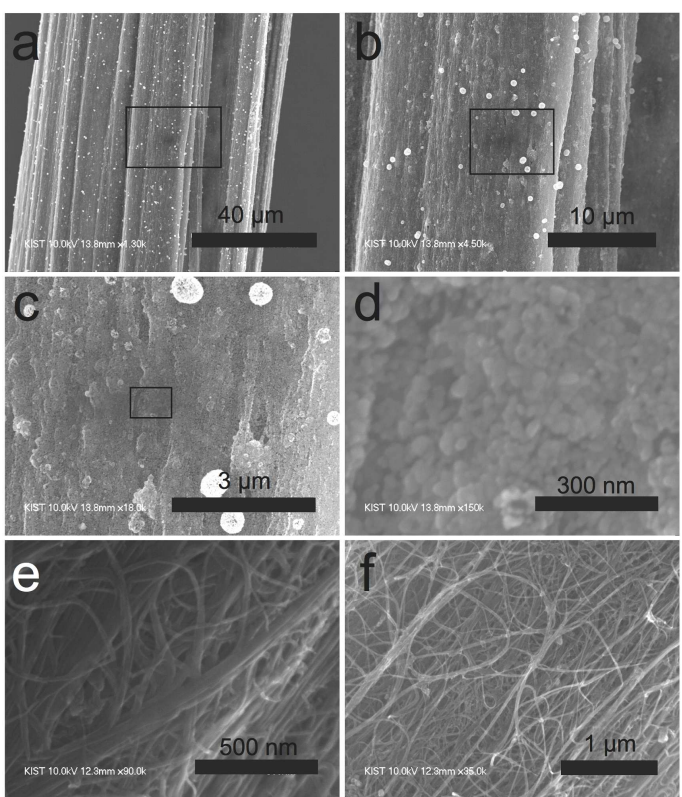

Fig. 2. SEM images of (a)-(d) ZnO-CNT fiber composites, (e) as-synthesized CNT fibers, and (f) CNT fibers after thermal and acid treatment, respectively. The $\mathrm{ZnO}$ nanoparticles were examined in greater detail by collecting magnified images of the rectangular regions shown in (a)-(c).

A synthetic scheme for obtaining the $\mathrm{ZnO}-\mathrm{CNT}$ fiber composites is illustrated in Fig. 3. As-synthesized CNT fibers (Fig. 3a) (or purified CNT fibers) were coated with $\mathrm{ZnO}$ acetate (Fig. 3b) and then heated at $450^{\circ} \mathrm{C}$ for $20 \mathrm{~min}$ to produce a $\mathrm{ZnO}-\mathrm{CNT}$ fiber composite in situ. Onto the CNT fibers, ZnO nanoparticles and aggregated spheres formed (Fig. 3c). SEM images of the resulting fibers are displayed in Fig. 3. As a control experiment $\mathrm{ZnO}$ nanoparticles were deposited onto CNT fibers ex situ (refer to Sect. 2). Figure 4a and b presents SEM images of the ex situ prepared fiber composites, indicating that $\mathrm{ZnO}$ nanoparticles were not deposited homogeneously as in the in situ process.

The atomic structure of the $\mathrm{ZnO}-\mathrm{CNT}$ fiber composite was analyzed by XRD. XRD spectra of the Si substrate, as-synthesized CNT fibers, $\mathrm{ZnO}-\mathrm{CNT}$ fiber composites and pure $\mathrm{ZnO}$ nanoparticles were obtained (Fig. 5). The spectrum of the as-synthesized CNT fibers (Fig. 5ab) displayed peaks at 19.07 and 25.10 (marked by arrows). The peak at 25.10 arose from crystalline carbon whereas the peak at 19.07 corresponded to higher $d$-spacings in the poorly crystalline carbon. Higher $d$-spacings may have resulted from oxygen containing functional groups that increased the carbon layer-layer distance. The peaks marked by black squares in curve $5 \mathrm{a} b$ were attributed 


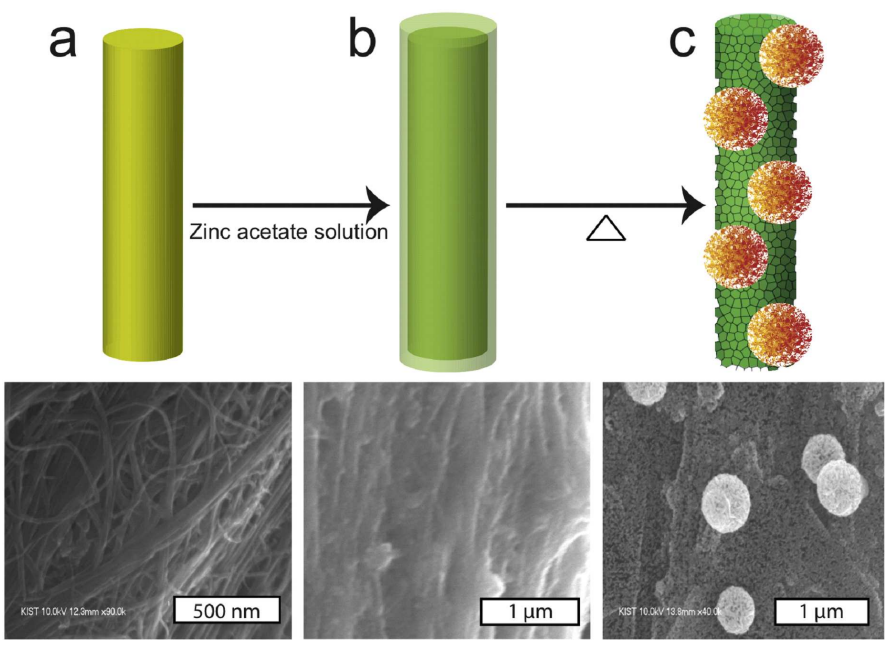

Fig. 3. Schematic diagram showing the fabrication of a $\mathrm{ZnO}-\mathrm{CNT}$ fiber composite in situ. (a) An assynthesized CNT fiber. (b) A zinc acetate-coated CNT fiber. (c) Thermal heating of the zinc acetate-coated CNT fibers led to the formation of the $\mathrm{ZnO}-\mathrm{CNT}$ fiber composite. SEM images of the outer surfaces at each step are displayed.
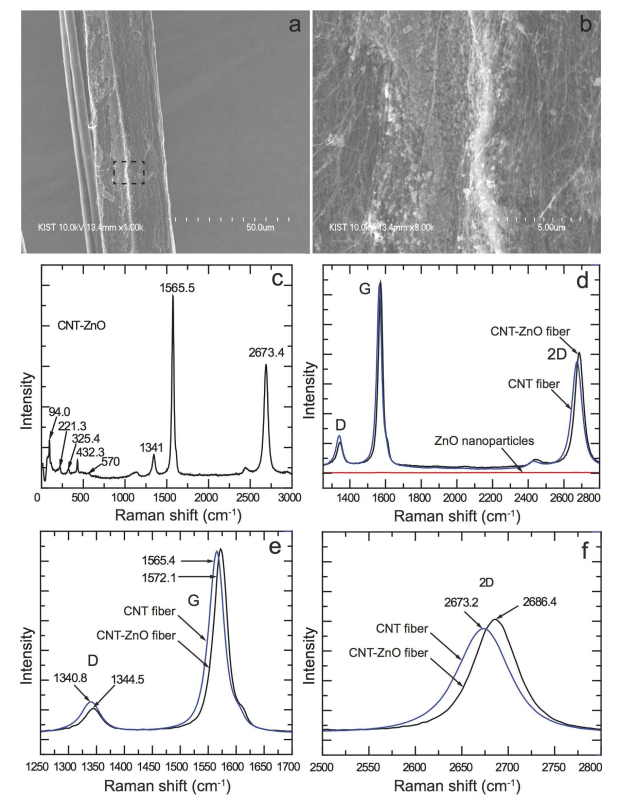

Fig. 4. (a) and (b) SEM images of the ex situ prepared $\mathrm{ZnO}-\mathrm{CNT}$ fiber composites. (c) Raman spectrum collected from the ex situ prepared $\mathrm{ZnO}-\mathrm{CNT}$ fiber composites. (d)-(f) Magnified spectra over the regions 1250$2800,1250-1700$, and $2500-2800 \mathrm{~cm}^{-1}$, respectively.

to an as-synthesized silicon substrate (curve 5a a). Curve $5 \mathrm{ac}$ corresponded to the XRD spectrum of a $\mathrm{ZnO}-\mathrm{CNT}$ fiber composite with carbon peaks at 16.03 and 25.10. The other peaks were attributed to the $\mathrm{ZnO}$ planes. All $\mathrm{ZnO}$ peaks were consistent with a hexagonal wurtzite structure in the $\mathrm{ZnO}$ nanoparticles of the JCPDS no.
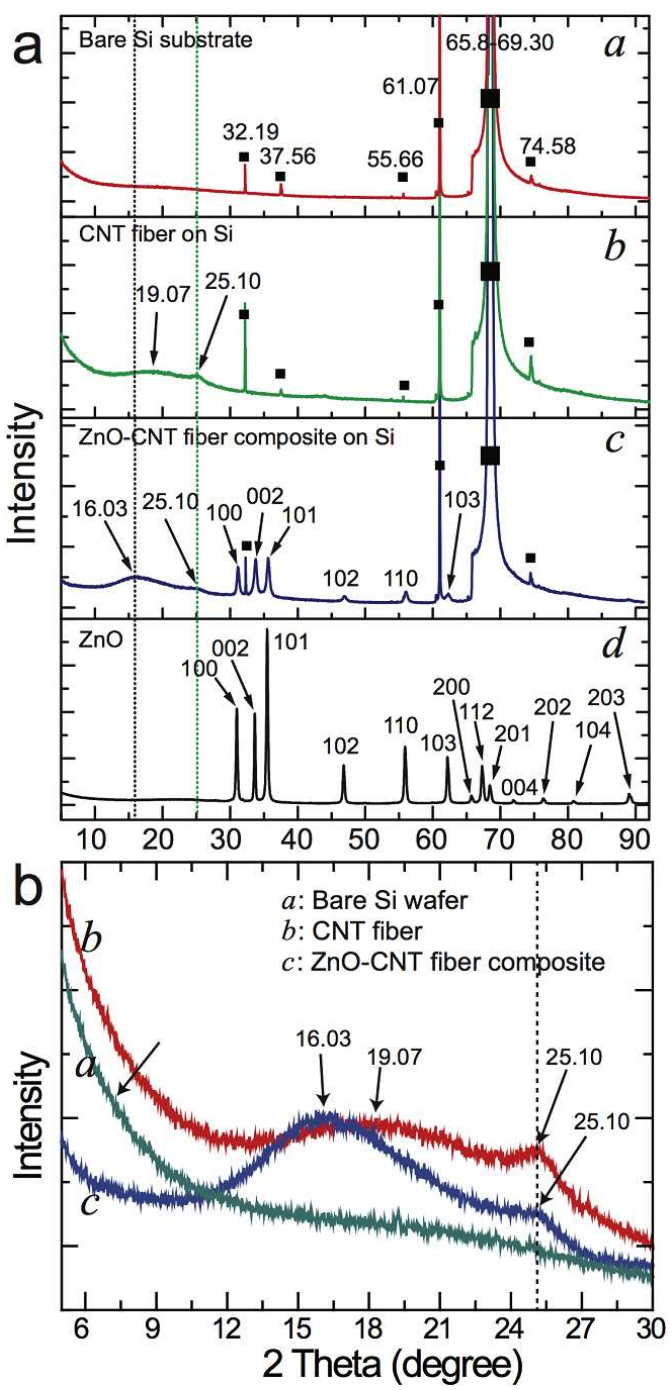

Fig. 5. (a) XRD spectra of $a$ bare Si wafer substrate, $b$ the as-synthesized CNT fiber on the Si wafer, $c$ the $\mathrm{ZnO}-\mathrm{CNT}$ fiber composite on the $\mathrm{Si}$ wafer, and $d$ the pure $\mathrm{ZnO}$ nanoparticles, respectively. (b) The peak position was elucidated by collecting magnified spectra over the region $5-30^{\circ}$ (curve $a$ ) for the Si wafer, (curve $b)$ for the CNT fibers on the Si wafer, and (curve $c$ ) for the $\mathrm{ZnO}-\mathrm{CNT}$ fiber composites on the $\mathrm{Si}$ wafer.

01-075-0576 [25]. The peaks corresponding to the 100, 002, 101, 102, and 103 planes of $\mathrm{ZnO}$ were observed in the XRD spectrum of the $\mathrm{ZnO}-\mathrm{CNT}$ fiber composite but peaks corresponding to the 200, 112, 201, 004, 202, 104, and 202 planes were not observed due to the high intensity peaks derived from the Si substrate. The XRD peaks corresponding to $\mathrm{ZnO}$ indicated that the $\mathrm{ZnO}$ nanoparticles of the composite assumed a hexagonal crystalline wurtzite structure.

The carbon peaks in curve $5(\mathrm{~b}) c$ were shifted to 16.03 from 19.07, indicating that the $\mathrm{ZnO}$ nanoparticles interacted with the poorly crystalline carbon. It is possible that $\mathrm{ZnO}$ intercalated between the walls of the multiwall 
CNTs or chemically bonded to the CNT surfaces through oxygen containing groups that formed $\mathrm{Zn}-\mathrm{O}-\mathrm{CNT}$ and $\mathrm{Zn}-\mathrm{OOC}-\mathrm{CNT}$ structures. Intercalation or chemical bonding was possible among the poorly crystalline carbon. The spectrum around the carbon peaks was magnified (panel 5(b)) to reveal that the poorly crystalline carbon peak shifted from 19.07 to 16.03 while the crystalline carbon peak (25.10) remained fixed in the as-synthesized CNT fibers and ZnO-CNT fiber composites.

The Raman spectra supported the presence of defect carbon atoms, which facilitated intercalation or chemical bonding between $\mathrm{ZnO}$ and the CNTs. The $D$ band in the Raman spectrum of the CNT fiber (Fig. 6a) indicated the presence of structural carbon defects in the CNT fibers. The $D$ band may have been due to the presence of oxygen containing functional groups or missing conjugation systems. The Raman peaks at $96.08,429.38$, and $561.2 \mathrm{~cm}^{-1}$ in curve $c$ corresponded to $E_{2}$ (low) [29], $E_{2}$ (high) [30], and $A 1-\mathrm{LO}$ (longitudinal optical) [29] phonons in the $\mathrm{ZnO}$ nanoparticles of the $\mathrm{ZnO}-\mathrm{CNT}$ fiber composite. These were also observed in curve $a$ for the pure $\mathrm{ZnO}$ nanoparticles. The peak at 221.6 was assigned to a second-order Raman intensity arising from the zone boundary ( $M$ point) phonon $2-E_{2}(M)$. This peak is normally found around 205-208 $\mathrm{cm}^{-1}[31,32]$, but in the $\mathrm{ZnO}-\mathrm{CNT}$ fiber composites it was blue-shifted due to the higher energy of $\mathrm{ZnO}$ as indicated by curve $a$. The peak at $322.4 \mathrm{~cm}^{-1}$ corresponded to a multiple phonon scattering process [32]. The $A 1-$ LO phonon peak $\left(561.2 \mathrm{~cm}^{-1}\right)$ was blue-shifted from $574,576 \mathrm{~cm}^{-1}$ [29], and $579 \mathrm{~cm}^{-1}$ [33], owing to the strong interaction between $\mathrm{ZnO}$ and the carbon atoms of the CNT fiber. The observation of a peak at $561.2 \mathrm{~cm}^{-1}$ corresponding to the $A 1-\mathrm{LO}$ mode was close to the theoretically predicted value $\left(548 \mathrm{~cm}^{-1}\right)$ [34].

Interestingly, a radial breathing mode (RBM) of the CNTs in the ZnO-CNT fiber composites was observed at $181.7 \mathrm{~cm}^{-1}$. This mode was not observed in the assynthesized CNTs (curve $b$ ). These results indicated that the RBM mode was activated by the interaction between the $\mathrm{ZnO}$ nanoparticles and the CNTs through chemical bonds which influenced the radial polarization of the CNTs. This interaction may occur if $\mathrm{ZnO}$ vibrates in the radial direction. The RBM is not readily enhanced by the surface deposition of $\mathrm{ZnO}$ nanoparticles without chemical bond formation.

Curve $c$ revealed that the $D, G$, and $2 D$ bands were blue-shifted by $3.7,6.7$, and $13.2 \mathrm{~cm}^{-1}$, respectively. These shifts were clearly observed in the magnified spectra (Fig. 4d-f) over the regions 1250-2800, 12501700 , and $2500-2800 \mathrm{~cm}^{-1}$, respectively. Similarly, the $\mathrm{ZnO}$ peaks in the $\mathrm{ZnO}-\mathrm{CNT}$ fiber composite revealed that the $E_{2}$ (high) and $A 1-\mathrm{LO}$ phonon modes shifted to 429.3 and $561.2 \mathrm{~cm}^{-1}$ (curve $c$ in Fig. 6b) from 432.3 and $570.0 \mathrm{~cm}^{-1}$ (curve $a$ in Fig. 6b), respectively. The vibrational shifts in the $D, G, 2 D, E_{2}$ (high), and $A 1-\mathrm{LO}$ phonon modes and the high-intensity RBM in the $\mathrm{ZnO}$ CNT fiber composite supported the presence of chemical

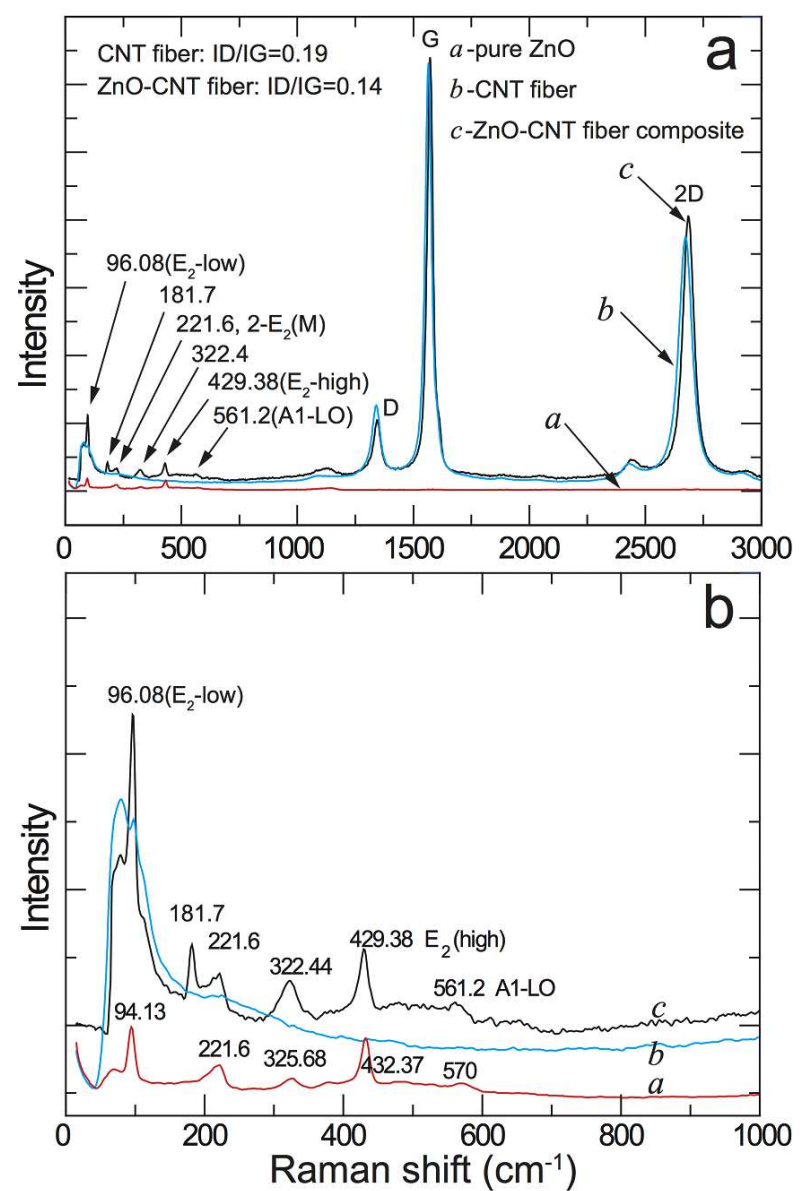

Fig. 6. (a) Raman spectra of (curve a) pure $\mathrm{ZnO}$, (curve b) CNT fibers, and (curve $c$ ) ZnO-CNT fiber composites, respectively. (b) The peak positions were elucidated by collecting the magnified spectra over the region $0-1000 \mathrm{~cm}^{-1}$ for (a).

bonds at the interface between the CNT fibers and the $\mathrm{ZnO}$ nanoparticles. The Raman spectrum was collected from the ex situ prepared $\mathrm{ZnO}-\mathrm{CNT}$ fiber composites (Fig. 4c). This spectrum presented no RBM and no significant shifts in the $D, G$, and $2 D$ bands of the CNTs in the $\mathrm{ZnO}-\mathrm{CNT}$ fiber composites. These features indicated a lack of significant interactions or chemical bonds at the interface between the CNTs and $\mathrm{ZnO}$ as a result of the ex situ process.

The inner sides of the $\mathrm{ZnO}-\mathrm{CNT}$ fiber composites were analyzed using FIB cuts. Figure 7a shows a crosssectional SEM image of a $\mathrm{ZnO}-\mathrm{CNT}$ fiber composite, revealing ribbon-like fibers with a folded edge. The upper region (marked by a dotted line, area-3) was damaged by the $\mathrm{Ga}$ ion beam. An SEM image taken from area- 2 is shown in Fig. 7b, revealing the presence of $\mathrm{ZnO}$ nanoparticles (marked by arrows) on the CNT surface. The rectangular region in Fig. $7 \mathrm{~b}$ is displayed in Fig. 7c and also reveals $\mathrm{ZnO}$ nanoparticles on the CNT fiber surface. The middle portion of the cross-sectional cut area- 1 is shown in Fig. 7d. This area reveals $\mathrm{ZnO}$ nanoparticles 
(some of them are marked by arrows). $\mathrm{ZnO}$ nanoparticles were also found in the folded portions of the $\mathrm{ZnO}-\mathrm{CNT}$ fiber composites (Fig. 7e). The inner side was analyzed by EDX mapping (Fig. 7f) to visualize the presence of $\mathrm{Zn}, \mathrm{O}$, and $\mathrm{C}$ elements. The inset of Fig. $7 \mathrm{f}$ was collected from the inner side and an analysis of the rectangular region revealed the presence of $\mathrm{ZnO}$ nanoparticles inside of the CNT fibers.

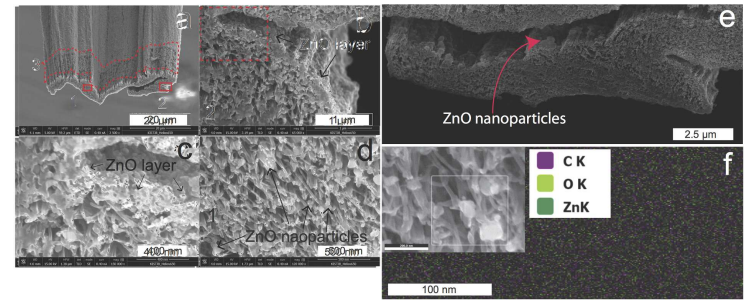

Fig. 7. (a) Cross-sectional cut of the $\mathrm{ZnO}-\mathrm{CNT}$ fiber composites obtained using the FIB technique. (b)(d) SEM images collected from the regions marked 2 (rectangle), 3 (dotted line), or 1 (rectangle) for (a). (e) SEM image of the folded part at the bottom of (a), revealing the presence of $\mathrm{ZnO}$ nanoparticles. (f) EDX mapping of the square region indicated in the inset of the SEM image of the $\mathrm{ZnO}-\mathrm{CNT}$ fiber composite. The $\mathrm{C}, \mathrm{O}$, and $\mathrm{Zn}$ elements in the mapping are indicated in purple, light green, and dark green, respectively.

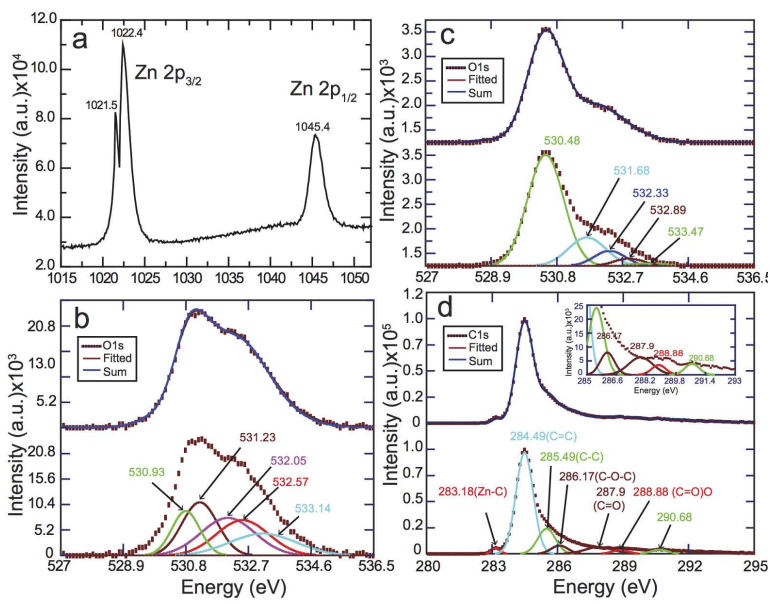

Fig. 8. XPS spectra of the $\mathrm{ZnO}-\mathrm{CNT}$ fiber composite for (a) $\mathrm{Zn}-2 p_{3 / 2}$ and $2 p_{1 / 2}$, (b) and (c) O-1s in the in situ- and ex situ-prepared samples, respectively, and (d) $\mathrm{C}-1 s$.

The characteristics of the $\mathrm{ZnO}$ bond with the CNT fibers were analyzed by XPS (Fig. 8). Peaks corresponding to $2 p_{3 / 2}$ and $2 p_{1 / 2}$ (Fig. 8 a) were observed at 1022.4 and $1045.4 \mathrm{eV}$, respectively, and were attributed to $\mathrm{Zn}$ in an oxidation state consistent with $\mathrm{ZnO}$. The shoulder peak at $1021.5 \mathrm{eV}$ for $\mathrm{Zn} 2 p_{3 / 2}$ suggested that the $\mathrm{ZnO}$ CNT fiber composite contained significant free nonoxidized $\mathrm{Zn}$ [35]. These results indicated that $\mathrm{ZnO}$ nanoparticles in the $\mathrm{ZnO}-\mathrm{CNT}$ fiber composite contained oxygen vacancy defects. The $\mathrm{O} 1 s$ peak (Fig. 8b) could be decom- posed into several components at 530.93, 531.23, 532.05, $532.57,533.14 \mathrm{eV}$ and their convolution fit well to the O1s curve (the upper curve). The low energy peak at $530.93 \mathrm{eV}$ corresponded to $\mathrm{O}^{-2}$ in the wurtzite structure of the $\mathrm{ZnO}$ lattice. The peaks at 531.23 and $532.05 \mathrm{eV}$ were related to $\mathrm{O}^{-2}$ moieties in the oxygen deficient region within the $\mathrm{ZnO}$ matrix [36]. The high-energy peak at $532.57 \mathrm{eV}$ originated from a $\mathrm{C}=\mathrm{O}$ functional group bonded to $\mathrm{Zn}$ in the poorly crystalline carbon atoms of CNT [37]. The highest energy peak at $533.14 \mathrm{eV}$ originated from a $\mathrm{C}-\mathrm{O}$ functional group [38]. Oxygen vacancy defects typically appear as one peak, but the $\mathrm{ZnO}-$ CNT fiber composite displayed two peaks at 531.23 and $532.05 \mathrm{eV}$. The higher electronegativity of carbon relative to $\mathrm{Zn}$ could play a role in providing two peaks. Carbon could be chemically bonded to $\mathrm{Zn}$ to form $\mathrm{C}-\mathrm{Zn}-\mathrm{C}$ $(531.23 \mathrm{eV})$ or $-\mathrm{O}-\mathrm{Zn}-\mathrm{C}(532.05 \mathrm{eV})$ groups at oxygen deficient sites. Thus the peaks observed at 532.57 and $533.14 \mathrm{eV}$ could have been due to the more electronegative oxygen environment [39]. Carbon could have been indirectly bonded to $\mathrm{Zn}$ to form $-\mathrm{O}-\mathrm{Zn}-\mathrm{O}-\mathrm{C}$ via the oxygen in $\mathrm{C}-\mathrm{O}$ or $\mathrm{C}=\mathrm{O}$ functional groups producing a more oxygen-rich environment. The peak at $531.23 \mathrm{eV}$ was more intense than the other peaks, indicating the creation of a significant number of oxygen vacancy defects in $\mathrm{ZnO}$.

The ex situ prepared $\mathrm{ZnO}-\mathrm{CNT}$ fiber composite was analyzed using XPS (Fig. 8c) and the peaks are summarized in Table I in comparison with the peaks obtained from the in situ prepared composite. The intensity of peaks 1 and $3-5$ in the ex situ composite were less intense than those obtained from the in situ composite. The binding energies for peaks 1, 3, and 4 were lower in the ex situ composite. The low intensity and low binding energy suggested a weaker interaction between $\mathrm{ZnO}$ and the CNTs in the ex situ $\mathrm{ZnO}-\mathrm{CNT}$ fiber composites. The intensity of peak 2 (due to the $\mathrm{C}-\mathrm{Zn}-\mathrm{C}$ bond) was insignificant in the ex situ composite, also due to the weak interactions between $\mathrm{ZnO}$ and carbon. Note that no RBM was observed in the Raman spectra (Fig. 4c) of the ex situ composite. These XPS features indicated the presence of a chemical interaction (or chemical bonding) in the in situ composite, consistent with the Raman analysis.

The deconvoluted curves of the C1s XPS spectra obtained from the $\mathrm{ZnO}-\mathrm{CNT}$ fiber composite are displayed in Fig. 6d. Several peaks corresponded to binding energies of $284.49,285.49,286.17,287.9,288.88$, and $290.68 \mathrm{eV}$ associated with the $\mathrm{C}=\mathrm{C}$ bond of the $s p^{2}$ carbon, the $\mathrm{C}-\mathrm{C}$ bond of the $s p^{3}$ amorphous carbon, $\mathrm{C}-\mathrm{O}-\mathrm{C}, \mathrm{C}=\mathrm{O}$, and $(\mathrm{C}=\mathrm{O}) \mathrm{O}$, respectively [40]. A $\pi-\pi^{*}$ transition in the $\mathrm{C}=\mathrm{C}$ network of the $\mathrm{ZnO}-\mathrm{CNT}$ fiber composite was observed at $290.68 \mathrm{eV}$. The low-energy peak at $283.18 \mathrm{eV}$ was attributed to a chemical bond between $\mathrm{Zn}$ and $\mathrm{C}$, for example, $\mathrm{Zn}-\mathrm{C}$ [41]. The peaks corresponding to $\mathrm{C} 1 \mathrm{~s}$ also supported the presence of oxygen containing functional groups in the CNTs of the ZnO-CNT fiber composite as in the Raman and O1s XPS spectra. 
The light absorption properties of the $\mathrm{ZnO}-\mathrm{CNT}$ fiber composite were investigated by measuring the UV-visible near-IR spectra (Fig. 9b). The $\mathrm{ZnO}-\mathrm{CNT}$ fiber composite (Fig. 9a) was carefully transferred to a quartz glass substrate (Fig. 9b). Pristine CNT fibers were prepared for comparison. The spectra of the $\mathrm{ZnO}-\mathrm{CNT}$ fiber composite and pristine CNT fibers are presented in Fig. 10a. Stronger light absorption was observed in the $\mathrm{ZnO}-\mathrm{CNT}$ fiber composite throughout the whole $300-2100 \mathrm{~nm}$ range as compared to the pristine CNT fiber. The ZnO absorption band in the composite was observed at $382 \mathrm{~nm}$, slightly higher than that of the pure $\mathrm{ZnO}$ (362 nm, Fig. 9c). The enhanced absorption of the $\mathrm{ZnO}-\mathrm{CNT}$ fiber composite may have been due to chemical bonds between the $\mathrm{Zn}$ and carbon [41], oxygen vacancy defects in the presence of the $\mathrm{Zn} d$ - $d$ transition [36], carbon doping of the $\mathrm{ZnO}$ nanoparticles [42], or multiple light scattering [43] by the $\mathrm{ZnO}$ nanospheres (as shown in Fig. 2).

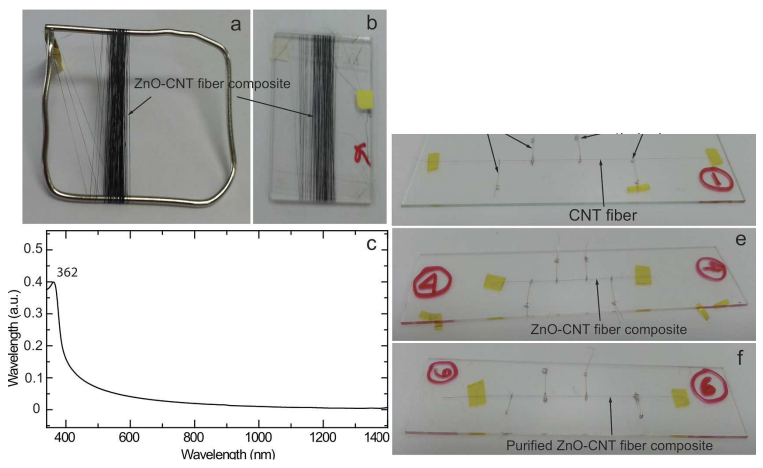

Fig. 9. (a) and (b) $\mathrm{ZnO}-\mathrm{CNT}$ fiber composite was transferred to a quartz glass substrate. (c) UV-visible near-IR spectra pure $\mathrm{ZnO}$. (d)-(f) The electrical conductivity CNT fiber, $\mathrm{ZnO}-\mathrm{CNT}$ fiber composite, and purified $\mathrm{ZnO}-\mathrm{CNT}$ fiber composite, respectively.

TABLE I

XPS O- $1 s$ peak positions, binding energies $E_{b}$, and intensities of the in situ and ex situ prepared $\mathrm{ZnO}-\mathrm{CNT}$.

\begin{tabular}{c|c|c|c|c}
\hline \hline \multirow{2}{*}{ Peak } & \multicolumn{2}{|c|}{ in situ } & \multicolumn{2}{c}{ ex situ } \\
\cline { 2 - 5 } & $E_{b}[\mathrm{eV}]$ & Intensity & $E_{b}[\mathrm{eV}]$ & Intensity \\
\hline 1 & 530.93 & $\approx 9000$ & 530.48 & 3500 \\
2 & 531.23 & 10500 & - & - \\
3 & 532.05 & 7700 & 531.68 & 1880 \\
4 & 532.57 & 7500 & 532.33 & 1600 \\
5 & 533.14 & 4300 & 533.47 & 1300
\end{tabular}

The effects of the $\mathrm{ZnO}$ nanoparticles on the thermal stabilities of the $\mathrm{ZnO}-\mathrm{CNT}$ fiber composites were tested using thermogravimetric analysis (TGA) at a heating rate of $10^{\circ} \mathrm{C} / \mathrm{min}$ under an $\mathrm{N}_{2}$ atmosphere. The TGA curves obtained from the $\mathrm{ZnO}-\mathrm{CNT}$ fiber composites, pristine CNT fibers, and $\mathrm{ZnO}$ nanoparticles are displayed in Fig. 7b. Significant thermal degradation of the pure $\mathrm{ZnO}$ was not observed until $880^{\circ} \mathrm{C}$, but the pristine CNT fibers slowly degraded from 100 to $540^{\circ} \mathrm{C}$. A dramatic degradation of the pristine CNT fibers occurred above $540^{\circ} \mathrm{C}$, and the CNT fibers were $74 \%$ degraded at $880^{\circ} \mathrm{C}$. On the other hand, no significant weight loss was observed in the $\mathrm{ZnO}-\mathrm{CNT}$ fiber composites over the range $100-540^{\circ} \mathrm{C}$. As the temperature increased from $540^{\circ} \mathrm{C}$ to $880^{\circ} \mathrm{C}$, slow weight loss was observed. A degradation of $26 \%$ occurred, corresponding to nearly one-third of the pristine CNT fibers. Our TGA analysis revealed that the prepared $\mathrm{ZnO}-\mathrm{CNT}$ fiber composite was more thermally stable than the pristine CNT fibers. ZnO nanoparticles on the CNT fiber surface could work as a protective layer against thermal decomposition.
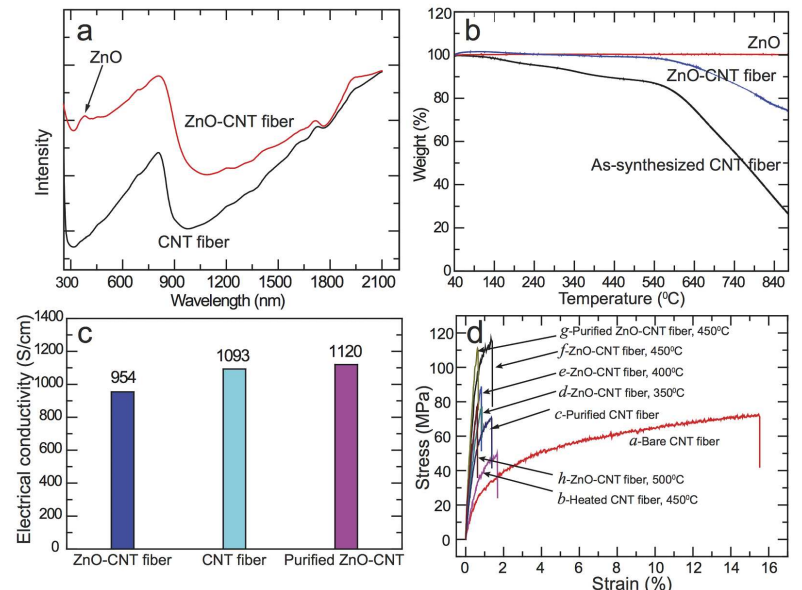

Fig. 10. (a) UV-vis NIR spectra of the as-synthesized CNTs and $\mathrm{ZnO}-\mathrm{CNT}$ fiber composites, respectively. (b) Thermogravimetric analysis of the pure $\mathrm{ZnO}$, CNT fibers, and $\mathrm{ZnO}-\mathrm{CNT}$ fiber composites, respectively. (c) Electrical conductivities of the as-synthesized CNTs and as-prepared and purified $\mathrm{ZnO}-\mathrm{CNT}$ fiber composites, respectively. (d) Mechanical properties (stress vs. strain curves) of the CNTs and $\mathrm{ZnO}-\mathrm{CNT}$ fiber composites prepared under various conditions. Curve $a-$ as-synthesized CNT fibers, curve $b-$ CNT fibers heated at $450{ }^{\circ} \mathrm{C}$ for 20 min, curve $c$ - purified CNT fibers, curves $d-h-\mathrm{ZnO}-\mathrm{CNT}$ fiber composites prepared at $350{ }^{\circ} \mathrm{C}, 400^{\circ} \mathrm{C}, 450{ }^{\circ} \mathrm{C}$, and $500^{\circ} \mathrm{C}$ for $20 \mathrm{~min}$, respectively, and curve $g$ - the purified $\mathrm{ZnO}-\mathrm{CNT}$ fiber composites prepared at $450{ }^{\circ} \mathrm{C}$.

The electrical conductivities of the $\mathrm{ZnO}-\mathrm{CNT}$ fiber composite and pristine CNT fibers were measured. Because $\mathrm{ZnO}$ is a semiconductor, a decrease in the electrical conductivity of the CNT fiber composite was expected. The measurement scheme is displayed in Fig. 9d9f. CNT fibers (Fig. 9d), ZnO-CNT fiber composites (Fig. 9e), and purified CNT composite fibers (Fig. 9f) were prepared on a glass slide using gold wires and silver paste. The sample was placed under a microscope to connect four needles (Fig. 11b) to the corresponding points (Fig. 11c) with the help of a microscope. A source meter and multimeter were used to measure the output voltage under application of a $105 \mu \mathrm{A}$ current to the source meter. The four points (marked 1-4 in Fig. 11c) of the CNT 

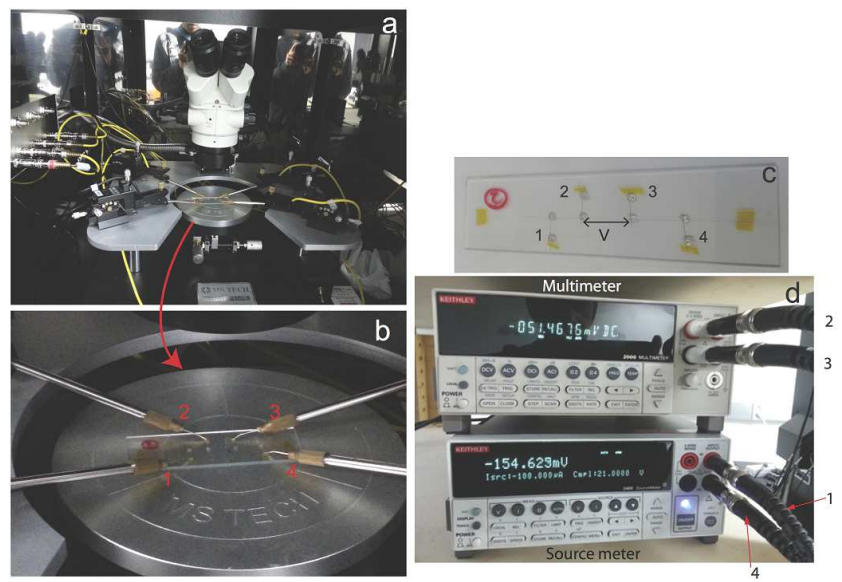

Fig. 11. (a)-(c) CNT fiber was placed under a microscope to connect four needles to the corresponding points with the help of a microscope. (d) A source meter and multimeter were used to measure the output voltage under application of a $105 \mu \mathrm{A}$ current to the source meter. The four points (marked 1-4 in (c)) of the CNT fiber were connected to the source meter and the multimeter's corresponding points (marked 1-4 in (d)). fiber were connected to the source meter and the multimeter's corresponding points (marked 1-4 in Fig. 11d). The output voltage between points 2 and 3 was converted to the corresponding resistance $(R)$, and the calculated resistance was converted to the resistivity $(\rho)$. The electrical conductivity was calculated to be $\sigma=1 / \rho=l / A R$, where $A$ is the cross-sectional area of the CNT fiber, and $l$ is the length of the CNT fiber between points 2 and 3 . The average electrical conductivities of the pristine CNT fibers (1093 S/cm), ZnO-CNT fiber composites $(954 \mathrm{~S} / \mathrm{cm})$, and purified $\mathrm{ZnO}-\mathrm{CNT}$ fiber composites $(1120 \mathrm{~S} / \mathrm{cm})$ were calculated (Fig. 10c). The electrical conductivity of the $\mathrm{ZnO}-\mathrm{CNT}$ fiber composite remained very high, indicating that the $\mathrm{ZnO}$ deposition did not significantly affect the conductivity due to direct and indirect chemical bonds between $\mathrm{Zn}$ and $\mathrm{C}$. Free $\mathrm{Zn}$ may form a conductive path between CNTs through the chemical bonds (Zn-C, C-Zn-C, O-Zn-C, Zn-O-C, or Zn-Zn-C) involving oxygen vacancies of $\mathrm{ZnO}$. The $\mathrm{ZnO}-\mathrm{CNT}$ fiber composite prepared using purified CNT fibers displayed a slightly enhanced electrical conductivity $(1120 \mathrm{~S} / \mathrm{cm})$ compared to that of the $\mathrm{ZnO}-\mathrm{CNT}$ composite and the pristine CNT fibers.

TABLE II

Mechanical properties of the CNTs and ZnO-CNT fiber composites prepared under various conditions.

\begin{tabular}{c|c|c|c|c}
\hline \hline Materials & Fabrication condition $(20 \mathrm{~min})$ & Strain [\%] & Stress [MPa] & Young modulus [GPa] \\
\hline pristine CNT fiber & no treatment & $16.6 \pm 2.2$ & $75.6 \pm 4.1$ & $4.7 \pm 0.6$ \\
ZnO-CNT fiber & $350^{\circ} \mathrm{C}$ & $1.43 \pm 1.07$ & $81.01 \pm 24.50$ & $12.17 \pm 3.31$ \\
ZnO-CNT fiber & $400^{\circ} \mathrm{C}$ & $1.2 \pm 1.0$ & $93.2 \pm 28.6$ & $15.6 \pm 3.85$ \\
ZnO-CNT fiber & $450^{\circ} \mathrm{C}$ & $1.09 \pm 0.27$ & $117.7 \pm 15.4$ & $19.8 \pm 4.1$ \\
ZnO-CNT fiber & $500^{\circ} \mathrm{C}$ & $0.52 \pm 0.19$ & $76.74 \pm 5.0$ & $21.6 \pm 3.5$ \\
purified CNT fiber & - & $1.2 \pm 0.4$ & $70.8 \pm 9.2$ & $9.2 \pm 3.5$ \\
purified ZnO-CNT fiber & $450^{\circ} \mathrm{C}$ & $0.65 \pm 0.30$ & $111.0 \pm 20.4$ & $21.2 \pm 3.2$ \\
pristine CNT fiber & $450^{\circ} \mathrm{C}$ & $1.19 \pm 0.50$ & $46.76 \pm 10.0$ & $6.59 \pm 1.1$
\end{tabular}

The mechanical properties of the $\mathrm{ZnO}-\mathrm{CNT}$ fiber composites produced at different temperatures $\left(30-500^{\circ} \mathrm{C}\right)$ were investigated (Fig. 10d and Table II) ZnO-CNT fiber composites prepared from $350-450{ }^{\circ} \mathrm{C}$ exhibited an enhanced Young modulus, which increased from 4.7 to 19.8 GPa and stress values that increased from 75.6 to $117.7 \mathrm{MPa}$. The $\mathrm{ZnO}-\mathrm{CNT}$ fiber composite prepared at $500^{\circ} \mathrm{C}$ displayed a slightly higher Young modulus $(21.6 \mathrm{GPa})$ although its stress $(76 \mathrm{MPa})$ was lower than that obtained at $450{ }^{\circ} \mathrm{C}$ (as-prepared). The pristine CNT fiber heated at $450{ }^{\circ} \mathrm{C}$ exhibited a low stress of $46.76 \mathrm{MPa}$ and a slight increase in the Young modulus (6.59 GPa) compared to the non-heated pristine CNT fibers. Purification of the CNTs prior to producing the composite did not improve the mechanical properties of the $\mathrm{ZnO}-\mathrm{CNT}$ fiber composite. This observation suggested that thermal heating of the purified CNTs did not significantly improve the mechanical properties of the $\mathrm{ZnO}-\mathrm{CNT}$ fiber composite. Chemical bonds between $\mathrm{Zn}$ and carbon were attributed to the enhanced mechanical properties of the $\mathrm{ZnO}-\mathrm{CNT}$ fiber composite.

\section{Conclusion}

The simple deposition of $\mathrm{ZnO}$ nanoparticles onto the CNT fiber surface via an in situ solution process provided a high electrical conductivity and a high Young modulus for the $\mathrm{ZnO}-\mathrm{CNT}$ fiber composites. A low temperature of $450^{\circ} \mathrm{C}$ was sufficient to prepare $\mathrm{ZnO}$ nanoparticles homogeneously deposited onto the CNT fiber surfaces as well as in the gaps of individual CNTs (inside of the CNT fibers). Strong interactions between the CNTs and ZnO nanoparticles were produced in the present in situ process, attributed to chemical bonds involving $\mathrm{Zn}$ and defect sites on the CNTs. The strong interactions enabled efficient thermal protection of the CNT fibers at temperatures up to $880^{\circ} \mathrm{C}$ and produced a conductive pathway that maintained the high electrical conductivity (954$1120 \mathrm{~S} / \mathrm{cm}$ ). An enhanced load bearing capacity and light absorption properties from the UV-visible to the near-IR regions could have resulted from oxygen vacancy defects and light scattering by the $\mathrm{ZnO}$ nanoparticles chemically bonded to the carbon atoms. 


\section{Acknowledgments}

This work was supported by grant from the Korean government (NRF-2015R1D1A1A-09059647). M.M.H. was supported by BK21 PLUS program.

\section{References}

[1] J. Bae, M.K. Song, Y.J. Park, J.M. Kim, M. Liu, Z.L. Wang, Angew. Chem. Int. Ed. 50, 1683 (2011).

[2] Y. Fu, X. Cai, H. Wu, Z. Lv, S. Hou, M. Peng, X. Yu, D. Zou, Adv. Mater. 24, 5713 (2012).

[3] S. Pan, Z. Yang, P. Chen, X. Fang, G. Guan, Z. Zhang, J. Deng, H. Peng, J. Phys. Chem. C 118, 16419 (2014).

[4] X. Xiao, T. Li, P. Yang, Y. Gao, H. Jin, W. Ni, W. Zhan, X. Zhang, Y. Cao, J. Zhong, L. Gong, W.-C. Yen, W. Mai, J. Chen, K. Huo, Y.-L. Chueh, Z.L. Wang, J. Zhou, ACS Nano 6, 9200 (2012).

[5] J. Ren, L. Li, C. Chen, X. Chen, Z. Cai, L. Qiu, Y. Wang, X. Zhu, H. Peng, Adv. Mater. 25, 1155 (2013).

[6] Y. Meng, Y. Zhao, C. Hu, H. Cheng, Y. Hu, Z. Zhang, G. Shi, L. Qu, Adv. Mater. 25, 2326 (2013).

[7] F. Vollrath, D.P. Knight, Nature 410, 541 (2001).

[8] Z. Xu, C. Gao, Macromolecules 43, 6716 (2010).

[9] W. Kylberg, F.A.D. Castro, P. Chabrecek, U. Sonderegger, B.T.T. Chu, F. Nüesch, R. Hany, Adv. Mater. 23, 1015 (2011).

[10] J.A. Lee, M.K. Shin, S.H. Kim, H.U. Cho, G.M. Spinks, G.G. Wallace, M.D. Lima, X. Lepró, M.E. Kozlov, R.H. Baughman, S.J. Kim, Nat. Commun. 4, 1970 (2013).

[11] K. Wang, Q. Meng, Y. Zhang, Z. Wei, M. Miao, Adv. Mater. 25, 1494 (2013).

[12] N. Behabtu, C.C. Young, D.E. Tsentalovich, O. Kleinerman, X. Wang, A.W.K. Ma, E.A. Bengio, R.F.T. Waarbeek, J.J.D. Jong, R.E. Hoogerwerf, S.B. Fairchild, J.B. Ferguson, B. Maruyama, J. Kono, Y. Talmon, Y. Cohen, M.J. Otto, M. Pasquali, Science 339, 182 (2013).

[13] H. Sun, X. You, J. Deng, X. Chen, Z. Yang, J. Ren, H. Peng, Adv. Mater. 26, 2868 (2014).

[14] H. Zhang, G. Cao, Z. Wang, Y. Yang, Z. Shi, Z. Gu, Nano Lett. 8, 2664 (2008).

[15] C. Yuan, X. Zhang, L. Su, B. Gao, L. Shen, J. Mater. Chem. 19, 5772 (2009).

[16] C.-C. Hu, K.-H. Chang, M.-C. Lin, Y.-T. Wu, Nano Lett. 6, 2690 (2006).

[17] Z. Chen, V. Augustyn, J. Wen, Y. Zhang, M. Shen, B. Dunn, Y. Lu, Adv. Mater. 23, 791 (2011).

[18] J.G. Ok, J.Y. Lee, H.W. Baac, S.H. Tawfick, L.J. Guo, A.J. Hart, ACS Appl. Mater. Interfaces 6, $874(2014)$.

[19] C.H. Hsu, H.Y. Liao, P.L. Kuo, J. Phys. Chem. C 114, 7933 (2010).
[20] B. Wu, D. Hu, Y. Kuang, B. Liu, X. Zhang, J. Chen, Angew. Chem. Int. Ed. 48, 4751 (2009).

[21] D. Wang, S. Lu, S.P. Jiang, Electrochim. Acta 55, 2964 (2010).

[22] E. Lorençon, A.S. Ferlauto, S.D. Oliveira, D.R. Miquita, R.R. Resende, R.G. Lacerda, L.O. Ladeira, ACS Appl. Mater. Interfaces 1, 2104 (2009).

[23] S. Guo, X. Pan, H. Gao, Z. Yang, J. Zhao, X. Bao, Chem. Eur. J. 16, 5379 (2010).

[24] A. Zamudio, A.L. Elías, J.A. Rodríguez-Manzo, F. López-Urías, G. Rodríguez-Gattorno, F. Lupo, M. Rühle, D.J. Smith, H. Terrones, D. Díaz, M. Terrones, Small 2, 346 (2006).

[25] M.M. Hossain, A.H.A. Mamun, J.R. Hahn, J. Phys. Chem. C 116, 23153 (2012).

[26] H. Shima, M.M. Hossain, J.R. Hahn, RSC Adv. 4, 41204 (2014).

[27] Y. Bu, Z. Chen, W. Li, B. Hou, ACS Appl. Mater. Interfaces 5, 12361 (2013).

[28] J. Lee, Y. Jung, J. Song, J.S. Kim, G.-W. Lee, H.J. Jeong, Y. Jeong, Carbon 50, 3889 (2012).

[29] B.H. Bairamov, A. Heinrich, G. Irmer, V.V. Toporov, E. Ziegler, Phys. Status Solidi B 119, 227 (1983).

[30] D.I. Son, B.W. Kwon, D.H. Park, W.S. Seo, Y. Yi, B. Angadi, C.-L. Lee, W.K. Choi, Nat. Nanotechnol. 7, 465 (2012).

[31] M. Rajalakshmi, A.K. Arora, B.S. Bendre, S. Mahamuni, J. Appl. Phys. 87, 2445 (2000).

[32] J.M. Calleja, M. Cardona, Phys. Rev. B 16, 3753 (1977).

[33] C.A. Arguello, D.L. Rousseau, S.P.S. Porto, Phys. Rev. 181, 1351 (1969).

[34] A. Zaoui, W. Sekkal, Phys. Rev. B 66, 174106 (2002).

[35] T. Ohshima, Y. Murakami, H. Kawasaki, Y. Suda, Y. Yagyu, Jpn. J. Appl. Phys. 50, 8S1 (2011).

[36] J. Wang, Z. Wang, B. Huang, Y. Ma, Y. Liu, X. Qin, X. Zhang, Y. Dai, ACS Appl. Mater. Interfaces 4 4024 (2012).

[37] Y. Lv, L. Yu, H. Huang, Y. Feng, D. Chen, X. Xie, Nanotechnology 23, 065402 (2012).

[38] M. Salavati-Niasari, F. Davar, M. Bazarganipour, Dalton Trans. 39, 7330 (2010).

[39] K. Jayanthi, M. Sunkara, S. Chawla, J. Phys. D Appl. Phys. 46, 325101 (2013).

[40] C.-C. Teng, C.-C.M. Ma, C.-H. Lu, S.-Y. Yang, S.-H. Lee, M.-C. Hsiao, M.-Y. Yen, K.-C. Chiou, T.-M. Lee, Carbon 49, 5107 (2011).

[41] Y.-P. Zhu, M. Li, Y.-L. Liu, T.-Z. Ren, Z.-Y. Yuan, J. Phys. Chem. C 118, 10963 (2014).

[42] X. Zhou, Y. Li, T. Peng, W. Xie, X. Zhao, Mater. Lett. 63, 1747 (2009).

[43] Q. Zhang, T.P. Chou, B. Russo, S.A. Jenekhe, G. Cao, Adv. Funct. Mater. 18, 1654 (2008). 